\title{
Stationary space-periodic structures with equal diffusion coefficients
}

\author{
Andresen, Peter Ragnar; Bache, Morten; Mosekilde, Erik; Dewel, G.; Borckmans, P.
}

Published in:

Physical Review E. Statistical, Nonlinear, and Soft Matter Physics

Link to article, DOI:

10.1103/PhysRevE.60.297

Publication date:

1999

Document Version

Publisher's PDF, also known as Version of record

Link back to DTU Orbit

Citation $(A P A)$ :

Andresen, P. R., Bache, M., Mosekilde, E., Dewel, G., \& Borckmans, P. (1999). Stationary space-periodic structures with equal diffusion coefficients. Physical Review E. Statistical, Nonlinear, and Soft Matter Physics, 60(1), 297-301. https://doi.org/10.1103/PhysRevE.60.297

\section{General rights}

Copyright and moral rights for the publications made accessible in the public portal are retained by the authors and/or other copyright owners and it is a condition of accessing publications that users recognise and abide by the legal requirements associated with these rights.

- Users may download and print one copy of any publication from the public portal for the purpose of private study or research.

- You may not further distribute the material or use it for any profit-making activity or commercial gain

- You may freely distribute the URL identifying the publication in the public portal 


\title{
Stationary space-periodic structures with equal diffusion coefficients
}

\author{
Peter Andresén,* Morten Bache, and Erik Mosekilde \\ Department of Physics, The Technical University of Denmark, DK-2800 Lyngby, Denmark
}

Guy Dewel and Pierre Borckmanns

Service de Chimie-Physique, Université Libre de Bruxelles, Code Postal 231, B-1050 Bruxelles, Belgium

(Received 13 July 1998; revised manuscript received 31 March 1999)

\begin{abstract}
The paper investigates a chemical reaction-diffusion model in an open flow system. It is shown that such a system may, with particular boundary conditions, exhibit stationary space-periodic structures even in the case of equal diffusion coefficients. This is confirmed through numerical simulations. [S1063-651X(99)11807-5]

PACS number(s): 82.40.Bj, 47.20.Ky
\end{abstract}

\section{INTRODUCTION}

One of the most well-known mechanisms for the formation of stationary space-periodic patterns is the diffusiondriven instability introduced by Alan Turing in his seminal paper from 1952 [1]. This symmetry-breaking instability is characterized by an intrinsic wavelength and therefore thought to be a good candidate for the mechanism behind biological pattern formation. The Turing instability applies to reaction-diffusion systems and relies on a difference of the diffusion coefficients in the system. The formation of stationary patterns through this instability may arise in dissipative chemical systems which contain an activator that stimulates its own production and an antagonist species, the inhibitor, that limits the growth of the activator [2]. The diffusion coefficient of the inhibitor must exceed that of the activator in order for an activated zone to be able to grow locally while lateral inhibition prevents its spreading and allows the formation of a concentration pattern. From an experimental point of view, the requirement of such a differential diffusion process has prevented direct verification of Turing patterns in aqueous solutions of small molecules and ions for many years. The experimental evidence for Turing structures in the chlorite-iodide-malonic acid (CIMA) reaction $[3,4]$ and variants hereof has only been made possible due to the reversible formation of a complex of low mobility involving the major activator species $\left(I^{-}\right)$and the color indicator used to visualize the system [5].

In chemical systems fresh reactants must continuously be supplied to the reactor in order to maintain a steady chemical pattern. In most experimental investigations of the diffusive chemical instability, gel reactors have been used to suppress the perturbative effect of the feeding flows on the chemical structures. Tubular reactors constitute another class of open chemical systems which may also exhibit nontrivial spatiotemporal behaviors. However, in the presence of such open flows one needs to distinguish between absolute and convective instabilities [6]. Absolute instability is characterized by any localized perturbation growing and eventually contaminating the entire system. In the case of a convective instability, on the other hand, localized perturbations are ad-

*Electronic address: andresen@chaos.fys.dtu.dk vected by the flow in such a way that they grow in the moving reference frame, but decay at any fixed position. The distinction between the absolutely and convectively unstable regions thus relies on whether the trailing front of the perturbation moves in the down or upstream direction. In the convectively unstable mode perturbations leave the system, and this mode can therefore only sustain a state different from the homogeneous unstable state in the presence of permanent boundary perturbations which break the Galilean invariance. Examples hereof are a time oscillating source (the signalling problem [6]), a noise source (leading to noise sustained structures [7]), or a constant displacement away from the homogeneous steady state of the system [8].

In this paper we develop an idea by Kuznetsov et al. [8] who showed how the formation of stationary periodic patterns may arise in an open flow system if the concentrations of the chemical species in the input flow are held at a constant value different from the homogeneous steady state and the flow velocity is sufficiently high. However, through their choice of values of the control parameters, Kuznetsov et al. considered the case with interacting Hopf and Turing instabilities which does give rise to a rich variety of structures, but which also tends to obscure the basic mechanism underlying the emergence of the above mentioned stationary space periodic structures. We therefore investigate this mechanism under a set of parameters which yield a clearer understanding, and show that it can indeed lead to the formation of such structures in the realistic case of equal diffusion coefficients.

\section{LINEAR STABILITY ANALYSIS}

As an example of a reaction-diffusion system which may lead to the formation of stationary periodic patterns we consider the Brusselator model in the presence of a constant flow ("plug flow"). If the chemical reactants are passive to the flow and the diffusion coefficients for the two chemical reactants are equal $\left(D_{U}=D_{V}\right)$ the corresponding reactiondiffusion-advection equations take the following form in one spatial dimension:

$$
\frac{\partial U}{\partial t}+\nu \frac{\partial U}{\partial x}=A-B U-U+U^{2} V+\frac{\partial^{2} U}{\partial x^{2}},
$$




$$
\frac{\partial V}{\partial t}+\nu \frac{\partial V}{\partial x}=B U-U^{2} V+\frac{\partial^{2} V}{\partial x^{2}}
$$

where $U(x, t)$ and $V(x, t)$ are the concentrations of the two chemical reactants, and $A$ and $B$ are control parameters.

This model obviously admits a simple homogeneous steady-state solution $U=U_{0}=A$ and $V=V_{0}=B / A$. Substituting

$$
U(x, t)=A+u(x, t), \quad V(x, t)=B / A+v(x, t)
$$

into Eqs. (1) and (2) we linearize the equations in order to determine the stability of the solution $\left(U_{0}, V_{0}\right)$. Due to the homogeneity in space and time we consider perturbations to be of the form $u(x, t)=\int u_{k, \omega} e^{i(k x-\omega t)} d k d \omega$ and similarly for $v(x, t)$. The relation between $k$ and $\omega$ is then given by the dispersion relation $D[k, \omega]=0$, and we note that the criterion for the homogeneous supercritical Hopf instability is

$$
B>B_{H}=1+A^{2}
$$

and that this is the first instability encountered as $B$ is increased in the case of equal diffusion coefficients.

The roots of the dispersion relation determine the stability of the system in the absence of flow. Traditionally the system is said to be unstable if the zeroes of the dispersion relation for some real wave number $k$ yield a complex solution $\omega(k)=\omega_{r}(k)+i \omega_{i}(k)$ with a positive imaginary part $\omega_{i}(k)$ $>0$ (temporal growth mode). In the presence of a flow, however, it is more useful to consider the solution $k(\omega)$ $=k_{r}(\omega)+i k_{i}(\omega)$ to the dispersion relation for real $\omega$ (spatial growth mode). Thus the system is unstable (allows spatially growing solutions) if there exists a root with negative imaginary part $k_{i}(\omega)<0$.

The physical interpretation of the roots of the dispersion relation and their behavior in $k$ space may now be determined through the Green's function formalism for a response to a small localized perturbation

$$
\begin{gathered}
{\left[\frac{\partial}{\partial t}+\nu \frac{\partial}{\partial x}-\frac{\partial^{2}}{\partial x^{2}}+1-B\right] u(x, t)-A^{2} v(x, t)=f(t) \delta(x),} \\
{\left[\frac{\partial}{\partial t}+\nu \frac{\partial}{\partial x}-\frac{\partial^{2}}{\partial x^{2}}+A^{2}\right] v(x, t)+B u(x, t)=g(t) \delta(x),}
\end{gathered}
$$

where $f(t)$ and $g(t)$ are the time-dependent behavior of the localized perturbations at the boundary $x=0$, and the perturbations are assumed to be switched on at $t=0$. By performing a Fourier transform the problem is restated in the spectral domain and the actual response in space and time may then be recovered by applying the inverse transformation

$$
\begin{aligned}
\{u(x, t) ; v(x, t)\}= & \frac{1}{(2 \pi)^{2}} \int_{L} d \omega \int_{M} d k \frac{\left\{I_{u}(k, \omega) ; I_{v}(k, \omega)\right\}}{D[k, \omega]} \\
& \times e^{i(k x-\omega t)},
\end{aligned}
$$

where the denominator $D[k, \omega]$ is given by

$$
\begin{aligned}
D[k, \omega]= & \left(-i \omega+i k \nu+k^{2}+1-B\right)\left(-i \omega+i k \nu+k^{2}+A^{2}\right) \\
& +A^{2} B
\end{aligned}
$$

and the numerators are, respectively,

$$
\begin{gathered}
I_{u}(k, \omega)=\left(-i \omega+i k \nu+k^{2}+A^{2}\right) F(\omega)+A^{2} G(\omega), \\
I_{v}(k, \omega)=\left(-i \omega+i k \nu+k^{2}+1-B\right) G(\omega)-B F(\omega) .
\end{gathered}
$$

Here $F(\omega)$ and $G(\omega)$ are Fourier transforms of the boundary perturbation

$$
\{F(\omega) ; G(\omega)\}=\int_{-\infty}^{\infty} d t e^{i \omega t}\{u(0, t) ; v(0, t)\}
$$

and are generally thought to be turned on for $t>0$.

The integration contour $L$ which is closed in the lower $\omega$ plane is chosen such that causality is fulfilled, i.e., $u(x, t)$ and $v(x, t)$ must be analytical above the integration contour in order for the response to be zero for $t<0$, and the $M$ contour runs along the real $k$ axis and is to be closed in the upper (lower) half $k$ plane for $x>0 \quad(x<0)$.

The inversion of the impulse response from the spectral domain into the space-time domain may be done through a straightforward residue integral ( $\omega$ integral) followed by an evaluation of the resulting residue integrals for large time through the method of steepest descent with $x / t$ fixed ( $k$ integral). In this evaluation great care must be taken in order to track the trajectories of the poles when the analytical continuation in the $k$ integral is performed. The integral thus consists of contributions arising from the poles of the dispersion relation $D[k, \omega]$ and from the poles of the boundary perturbation $[F(\omega)$ and $G(\omega)]$. In the convectively unstable state it is the poles of the boundary perturbation which yield the dominating contributions to the impulse response.

The transition of the unstable state from absolute to convective instability takes place at a critical velocity $\nu_{c}$. This velocity may be determined by considering the long time behavior of the wave number $k_{0}$ with zero group velocity [6]

$$
\left.\frac{d \omega(k)}{d k}\right|_{k=k_{0}}=0
$$

The transition from the absolute to convective unstable case then occurs when the temporal growth rate of this wave number is zero, i.e., $\omega_{i}\left(k_{0}\right)=0$. For the Brusselator in the unstable Hopf mode this yields

$$
\nu_{c}=\sqrt{2\left(B-B_{H}\right)} .
$$

In the absolutely unstable state any small local perturbation will lead to the whole system becoming contaminated by the bifurcated state (here, the Hopf state). Hence, apart from a small boundary layer at the inlet, a permanent perturbation will not affect the overall structure of the system. In the convectively unstable state a single small local perturbation will grow while being advected by the flow. It will thus eventually leave the boundaries of the system, and the system will return to the homogenous steady state. However, if the system is continuously perturbed it will not be able to 
return to the homogenous steady state and a new state will be established which is sustained by the boundary perturbation.

\section{BOUNDARY PERTURBATIONS}

Now consider a boundary perturbation in the convectively unstable state which consists of a constant displacement from the homogeneous equilibrium value of one or both of the chemical species $\left(U_{0}\right)$ at the inlet $(x=0)$. In the case of only one of the species being displaced we have

$$
\{f(t), g(t)\}=\{\epsilon H(t), 0\},
$$

where $\epsilon>0, H(t)$ is the Heaviside function, and therefore

$$
\{F(\omega), G(\omega)\}=\{2 \pi \delta(\omega), 0\} .
$$

The inverse transformation of the impulse response now yields three terms for the residue-integral in $\omega$; two from the quadratic dispersion relation $D[k, \omega]=0$ and one from the perturbation $(\omega=0)$. It is the contribution from the perturbation which dominates for long times when the system is convectively unstable [6] and thus the integration over $k$ space for this pole determines the response. As the integration contour $M$ [in Eq. (6)] is closed in the upper half $k$ plane for $x>0$, only poles which cross the real $k$ axis and enter the lower half plane as $\nu$ is increased yield spatially amplifying contributions. The condition for such contributions may thus be found by looking for pure real wave number $\left(k_{i}=0\right)$ solutions of the dispersion relation Eq. (7) $(\omega=0)$. Separating the real and imaginary part yields the following condition for the flow velocity:

$$
\nu_{T}=\sqrt{\frac{4 A^{2}-\left(B-B_{H}\right)^{2}}{2\left(B-B_{H}\right)}} .
$$

For flow velocities above this value the impulse response due to the boundary perturbation at the inlet will yield a solution with negative $k_{i}$ thus giving rise to stationary $(\omega=0)$ spatially amplifying waves. As the system is considered to be in the convectively unstable state the response arising from the zeroes of the dispersion relation (i.e., the underlying unstable Hopf state) will be flushed out of the system leaving only the above stationary state to dominate the system. Note that if the perturbation had not been permanent, thus continuously giving rise to the spatially growing modes, the system would just have returned to its equilibrium value. Thus for flow rates $\nu>\nu_{T}$ and $\nu>\nu_{c}$ (see Fig. 1) a permanent boundary perturbation can generate stationary chemical patterns in the entire reactor even in systems with equal diffusion coefficients. Such a global structure provides a further example of the sensitivity of convectively unstable states to external perturbations. Previously, Ortoleva and Ross [9] have reported an example of penetration of boundary perturbations in a chemical system which gives rise to stationary structures. They, however, do not presuppose the existence of a flow and therefore their mechanism for the emergence of global patterns is only valid at marginal stability (i.e., at the exact bifurcation point, in our case for the Brusselator $B=B_{H}$ ).

Numerical simulations of Eqs. (1) and (2) using a CrankNicholson semi-implicit scheme were performed in order to verify our predictions. With the flow running from the left to

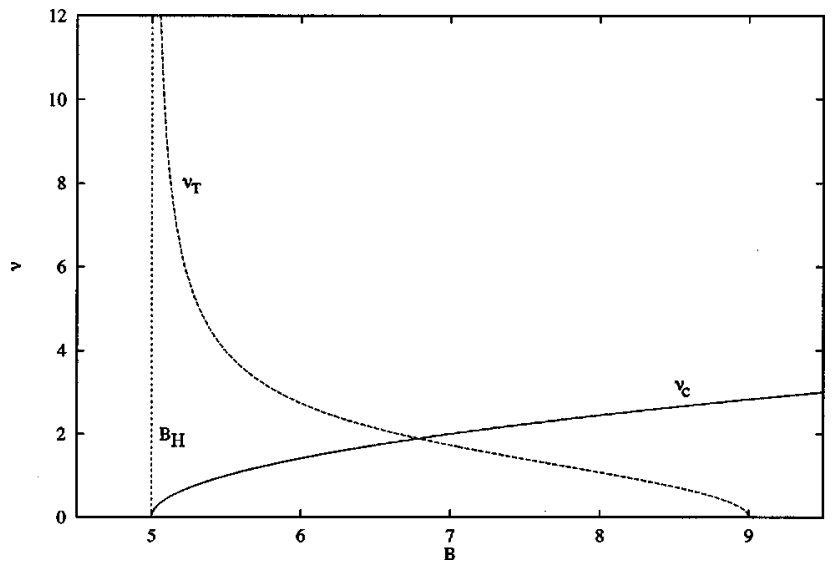

FIG. 1. Bifurcation diagram for the Brusselator with flow. Shown is the Hopf bifurcation curve $\left(B_{H}\right)$ which for $A=2$ occurs at $B=B_{H}=5.0$, as well as the threshold of the absolute-convective instability $\left(\nu_{c}\right)$ and the transition to spatially amplifying waves $\left(\nu_{T}\right)$ in the presence of a constant boundary perturbation. Note that stationary space-periodic structures may thus only occur in the upper right hand region, above both $\nu_{c}$ and $\nu_{T}$.

the right the boundary perturbation was applied to the left edge while no-flux boundary conditions were applied at the right edge. The system was initialized in the steady state at parameter values of $A$ and $B$ which were above the Hopf instability. Figure 2 shows how the stationary space-periodic pattern invades the homogeneous steady state in accordance with our predictions. Similarily to the Turing instability the pattern arises through a spatial decoupling of the two chemical species as shown in Fig. 3. Although the instability pertains to the allowance of spatially growing waves, the growth will be limited by nonlinear effects, and the amplitude will thus saturate as shown in Fig. 4. The amplitude and wave number of the resulting structure are intrinsic. In particular the saturated amplitude as well as selected wave number of the structure is independent of whether one or both chemical species are perturbed at the inlet. Furthermore the size of the perturbation is also irrelevant as is illustrated in Fig. 4. Even perturbations as small as $O\left(10^{-4}\right)$ lead to spatially growing waves which is to be expected since the system is in an unstable state, and all perturbations lead to the same saturated amplitude. The perturbation at the inlet thus induces a spatial phase shift much in the same manner as the Hopf state comes about by a temporal phase shift.

The selected wave number of the stationary spaceperiodic structures may be determined by noting that in order for the emerging pattern to be stationary in the laboratory frame the phase velocity of the waves propagating in the opposite direction of the flow must satisfy the condition

$$
\frac{\omega(k)}{k}=\nu
$$

(a condition reminiscent of the requirement for the "Cherenkov" effect to emerge in special relativity [11]). Note that only the Hopf instability gives rise to such an effect, as it allows traveling wave solutions. Weakly nonlinear theory yields the complex Ginzburg-Landau equation as the reduced description of the Brusselator close to the Hopf bifurcation. 


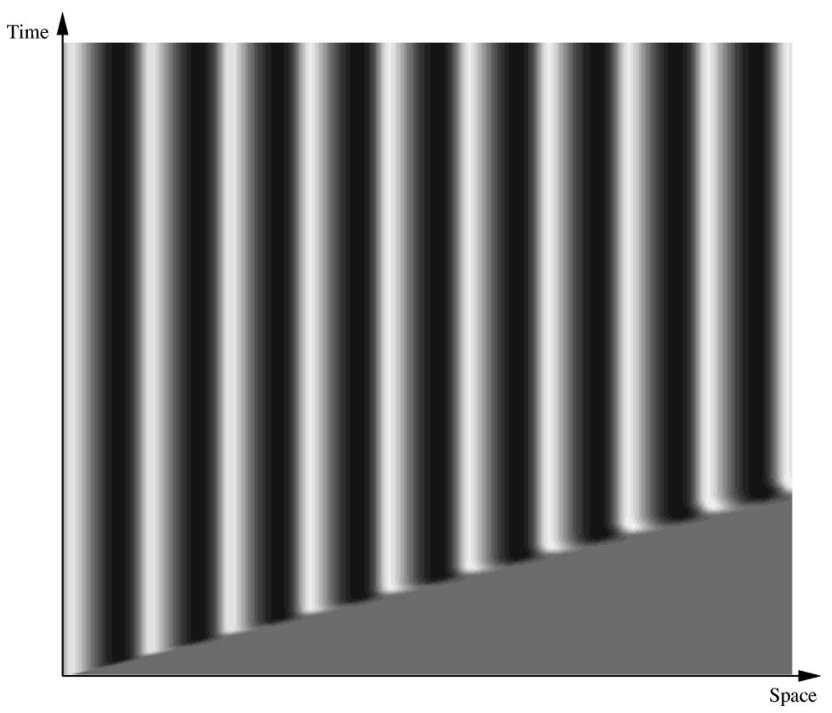

FIG. 2. Space-time plot of a simulation showing stationary space-periodic structures due to a boundary perturbation at the left edge $(A=2, B=5.2$, and $\nu=10.0)$. Black signifies a low concentration of species $U$ while white signifies a high concentration. The grey triangle in the lower right corner represents the homogeneous steady state. The structure generated by the boundary perturbation is not the underlying Hopf mode, but rather a structure with an intrinsic wave number. The system has a width of 300 and the simulation was run for 100 time units with space and time resolutions of $\Delta x=0.1$ and $\Delta t=0.01$, respectively.

We may thus utilize the dispersion relation of the complex Ginzburg-Landau equation in order to calculate the selected wave number. Thus inserting

$$
\omega(k)=\omega_{c}+\left(k^{2}-\frac{B-B_{H}}{2}\right)\left(\frac{4 A^{4}-7 A^{2}+4}{3 A\left(A^{2}+2\right)}\right),
$$

where $\omega_{c}=A$ is the natural Hopf oscillation frequency, in Eq. (11) yields

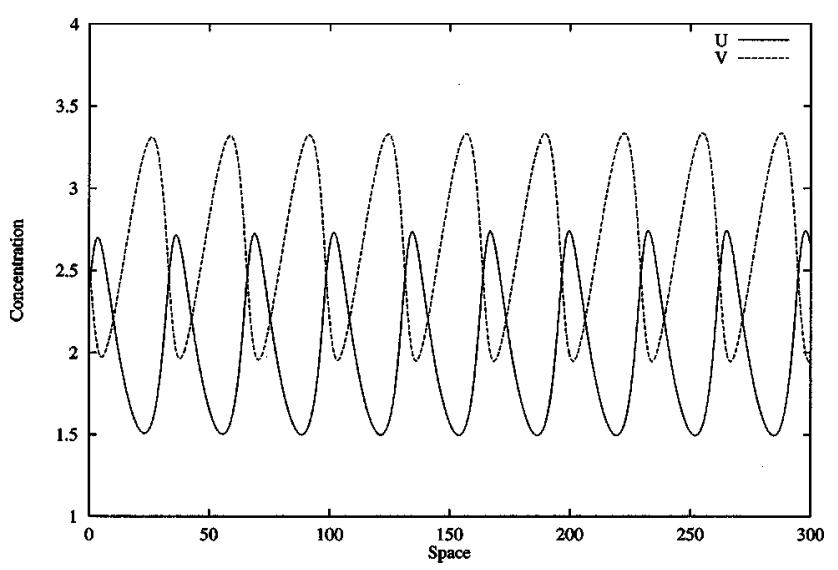

FIG. 3. The concentrations of the two chemical reactants $U$ and $V$ shown as functions of space (same parameter values as in Fig. 2).The boundary perturbation along with the applied flow leads to a spatial phase shift thus inducing a local decoupling of the reactants. The apparent decrease in the amplitude of the concentrations is a transient effect, and the amplitude is indeed stable.

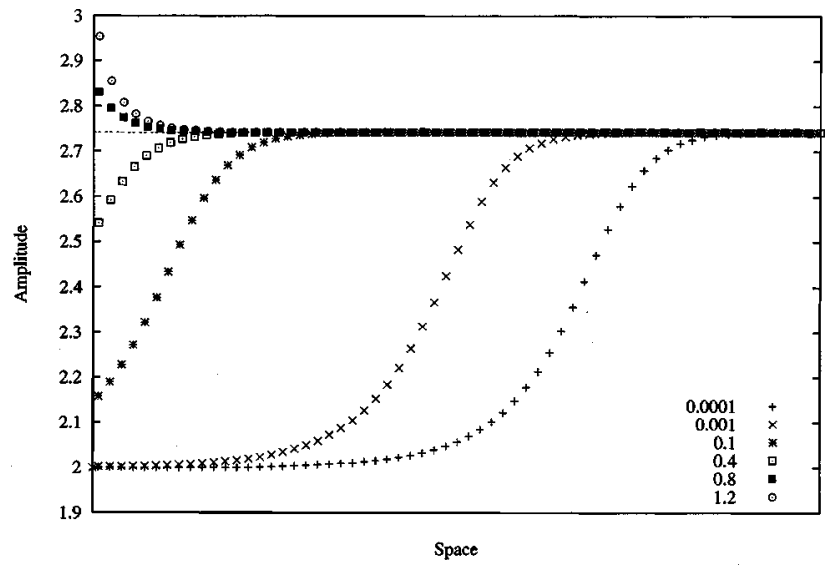

FIG. 4. The relaxation of the amplitude for the species $U$ of the resulting structure for different sizes of the stationary boundary perturbation $-10^{-4}, 10^{-3}, 0.1,0.4,0.8$, and 1.2 . The saturated amplitude has a value of $2.742(A=2.0, B=5.2$, and $\nu=9.0)$.

$$
\begin{aligned}
k= & \frac{3 A\left(A^{2}+2\right)}{2\left(4 A^{4}-7 A^{2}+4\right)} \\
& \times\left[\nu-\sqrt{\nu^{2}-4\left(\omega_{c}-\frac{B-B_{H}}{2} \frac{4 A^{4}-7 A^{2}+4}{3 A\left(A^{2}+2\right)}\right)} .\right.
\end{aligned}
$$

The selected wave number decreases with increasing velocity as is illustrated in Fig. 5 and the measured wave numbers are in good agreement with the theoretically predicted ones.

Another possible boundary perturbation would be to apply a periodic varying perturbation at the inlet of the system. However, such a perturbation would not allow stationary space-periodic waves to emerge, which may easily be verified through an analysis of the impulse response. The boundary perturbation would in this case introduce a forcing frequency $\omega_{f}$ into the problem yielding

$$
\{f(t), g(t)\}=\left\{\epsilon e^{i \omega_{f} t} H(t), \epsilon e^{i \omega_{f} t} H(t)\right\},
$$

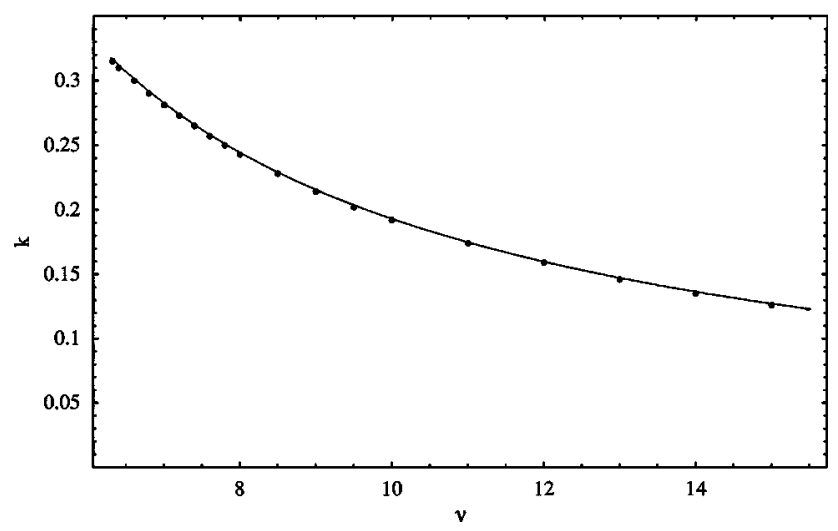

FIG. 5. Measurements ( $)$ of the selected wave number for the boundary perturbations compared to the theoretical prediction according to Eq. (11) $\left(A=2.0, B=5.2, \nu_{T}=6.317\right)$. The selected wave number of the stationary space-periodic structure decreases with increasing flow velocity. 


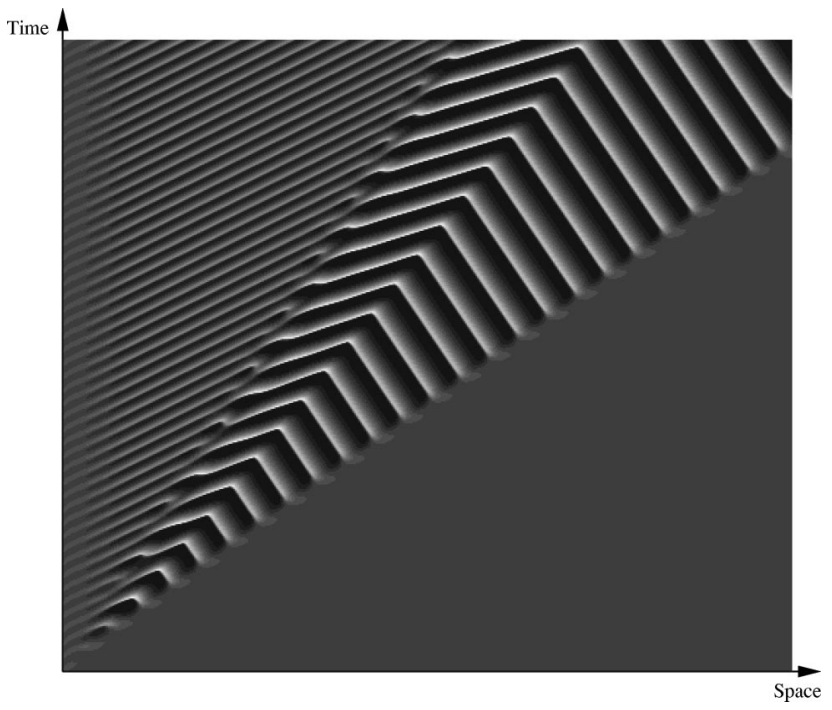

FIG. 6. Space-time plot showing how the periodic boundary perturbation at the inlet (to the left of the figure) penetrates into system $\left(A=2.0, B=6.0, \nu=2.3\right.$, and $\left.\omega_{f}=3.0\right)$. The frequency of the signal at a fixed position in space has been determined to be exactly $\omega_{f}$. Note that the structure which appears to the right of the penetrating signal is the initial response (the Hopf mode) which is advected out of the system due to the convective instability. The grey area in the lower right part is the homogeneous steady state. The simulation parameters are the same as in Fig. 2.

where $\epsilon>0$ and the integral which determines the behavior of the system in the convective unstable state would then always yield a time-dependent solution with frequency $\omega_{f}$. This type of behavior is illustrated in Fig. 6 where it is seen how a permanent oscillating perturbation at the left boundary penetrates into the system giving rise to a structure which has a frequency of exactly $\omega_{f}$, while the Hopf mode is advected out of the system. Neither would a permanent noise source applied to the boundary give rise to stationary spaceperiodic structures. This case has been studied by Deissler
[7] for the complex Ginzburg-Landau equation, and he notes that such a perturbation, if applied in the convectively unstable state, will lead to the system exhibiting a selective spatial amplification of the noise resulting in spatially growing waves. The resulting state of the system is thus termed a noise-sustained structure.

The pattern forming mechanism discussed in the present paper should not be confused with the differential flow induced chemical instability (DIFICI) [10] which gives rise to traveling waves when the difference between the flow rates of the activator and the inhibitor exceeds a critical value. For the patterns considered here the flow rate and the boundary perturbation together induce a spatial phase shift between the counteracting species $U$ and $V$ as shown in Fig. 3. As in the case of the Turing instability and DIFICI it is precisely the local decoupling that allows for the onset of spatial pattern formation.

\section{CONCLUSION}

In conclusion, we have shown that a permanent boundary perturbation can create a global steady periodic pattern in the state that is convectively unstable with respect to the Hopf mode. The only requirement is that the flow velocity is greater than $\nu_{T}$ [Eq. (10)]. Interestingly enough this may happen even for equal diffusion coefficients which prohibits the formation of Turing patterns due to the requirement of a local decoupling of the interacting species. Because it is submitted to less stringent requirements than the Turing or the DIFICI instabilities an experimental verification of the effect predicted above should be possible. An example may already have been provided in Ref. [12].

\section{ACKNOWLEDGMENTS}

P.A. and M.B. wish to thank Professor G. Nicolis for his kind hospitality. M.B. acknowledges the support of the ERASMUS program. G.D. and P.B. received support from the FNRS (Belgium).
[1] A.M. Turing, Philos. Trans. R. Soc. London, Ser. B 327, 37 (1952).

[2] G. Nicolis and I. Prigogine, Self-Organization in Nonequilibrium Chemical Systems (Wiley, New York, 1977).

[3] V. Castets, E. Dulos, J. Boissonade, and P. De Kepper, Phys. Rev. Lett. 64, 2953 (1990).

[4] Q. Ouyang and H.L. Swinney, Nature (London) 352, 610 (1991).

[5] I. Lengyel and I.R. Epstein, Proc. Natl. Acad. Sci. USA 89, 3977 (1992).

[6] P. Huerre, in Instabilities and Nonequilibrium Structures, ed- ited by E. Tirapegui and D. Villarroel (Reidel, 1987), pp. 141177.

[7] R.J. Deissler, J. Stat. Phys. 40, 371 (1985).

[8] S.P. Kuznetsov, E. Mosekilde, G. Dewel, and P. Borckmanns, J. Chem. Phys. 106, 7609 (1997).

[9] P.J. Ortoleva and J. Ross, J. Chem. Phys. 56, 287 (1972).

[10] A.B. Rovinsky and M. Menzinger, Phys. Rev. Lett. 69, 1193 (1992); 70, 778 (1993).

[11] L. D. Landau and E. M. Lifshitz, Electrodynamics of Continous Media (Pergamon, Oxford, 1984).

[12] M. Marek and E. Svobodova, Biophys. Chem. 2, 263 (1975). 